\title{
Approaches for Promoting Accurate Atom Probe Reconstruction
}

\author{
T.J. Prosa ${ }^{1}$, B.P. Geiser ${ }^{1}$, D. Reinhard ${ }^{1}$, Y. Chen ${ }^{1}$ and D.J. Larson ${ }^{1}$ \\ ${ }^{1 .}$ CAMECA Instruments Inc., Madison, WI, USA
}

Comparison of atom probe tomography (APT) results from different instruments, different users, or different samples requires confidence in overall reconstruction accuracy. The conventional reconstruction algorithm utilizes a single point-projection onto a spherical surface [1,2]. Although there are a limited number of parameters used to prescribe how detector events are converted to a 3D set of reconstructed coordinates, some constraints are necessary to ensure minimal precision and accuracy. Key inputs in the reconstruction algorithm used in the commercially available IVAS software (CAMECA Instruments, Inc.) include ionic volume, detection efficiency, image compression factor (ICF), either tip radius (initial or final) or the product of geometrical field factor (k) and evaporation field (F), sphere-to-cone ratio, and radial evolution procedure (voltage, shank angle, or tip profile) [3]. When quality protocols are implemented, reliable comparisons can be made [4]. The available approaches for properly choosing reconstruction parameters will be the focus of this presentation.

Some homogeneous materials exhibit highly ordered evaporation. Fig. 1 shows a single crystal, pure nickel, field-desorption map where the poles can be easily identified and the atomic planes are readily apparent in the reconstruction. For these types of systems, the crystallography serves as an internal calibration standard for constraining the reconstruction parameters [5]. A spatial distribution map (SDM) can be calculated to extract atomic-spacing information for feedback to the reconstruction to promote high accuracy [6].

There are homogeneous materials where no short-range order or crystallographic features are observed for use as internal calibration standards. Fig. 2 shows reconstruction of a zircon APT analysis. SEM measurements of the APT tip before and after analysis are required to provide information (analysis depth, final tip radius, shank evolution, sphere-to-cone ratio) to promote accurate reconstruction.

Finally, there are material systems where the single point-projection approach obviously fails to accurately reconstruct all the known features simultaneously. Fig. 3 shows the reconstruction for a multi-layer LED quantum-well device structure where each layer is known to be flat and parallel to its neighbors. It is not possible to choose a single set of reconstruction parameters that provides accurate reconstruction for the entire analysis volume. Some post-reconstruction corrections exist to reposition atoms and improve accuracy. Fig. 3 highlights the Landmark Reconstruction approach (similar to [7]). Chemical interfaces are identified and the positions then scaled to force the interfaces to become flat. These scaled positions are then propagated through the entire depth of the reconstruction.

References:

[1] P. Bas et al, Appl. Surf. Sci. 87/88 (1995) p. 298.

[2] B.P. Geiser et al, Microsc. Microanal. 15 (S2) (2009) p. 292.

[3] D.J. Larson et al in "Local Electrode Atom Probe Tomography", (Springer, New York, 2013).

[4] T.J. Prosa et al, Ultramicroscopy 132 (2013) p. 179.

[5] B. Gault et al, Microsc. Microanal. 14 (2008) p. 296.

[6] B.P. Geiser et al, Microsc. Microanal. 13 (2007) p. 437.

[7] F. Vurpillot et al, Ultramicroscopy 132 (2013) p. 19. 

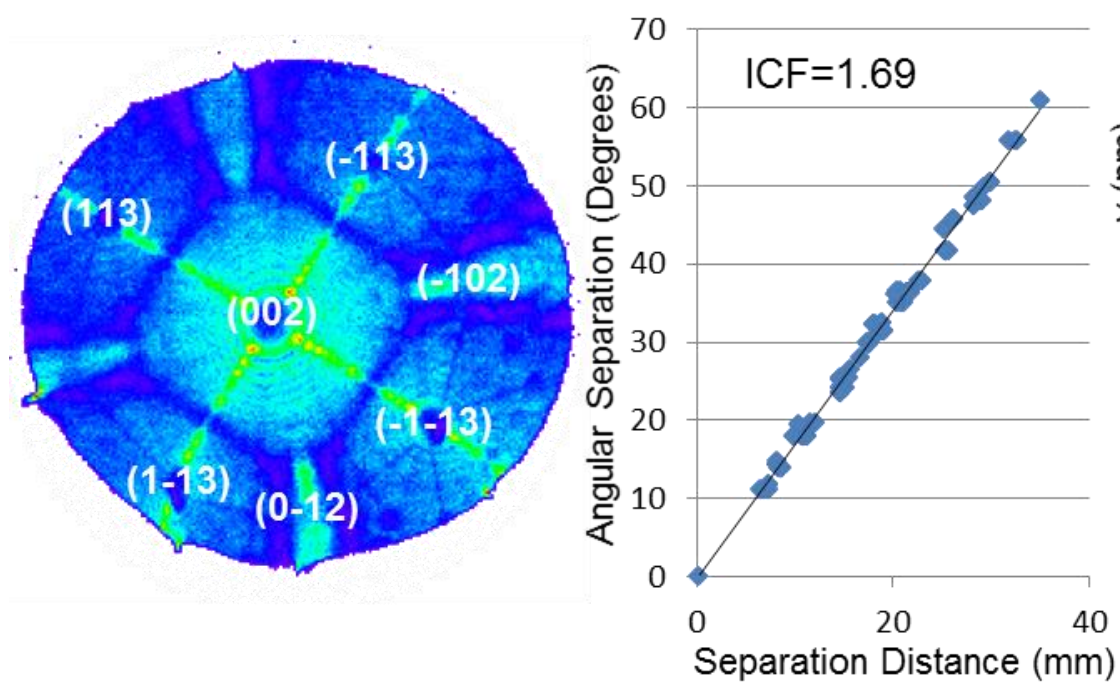

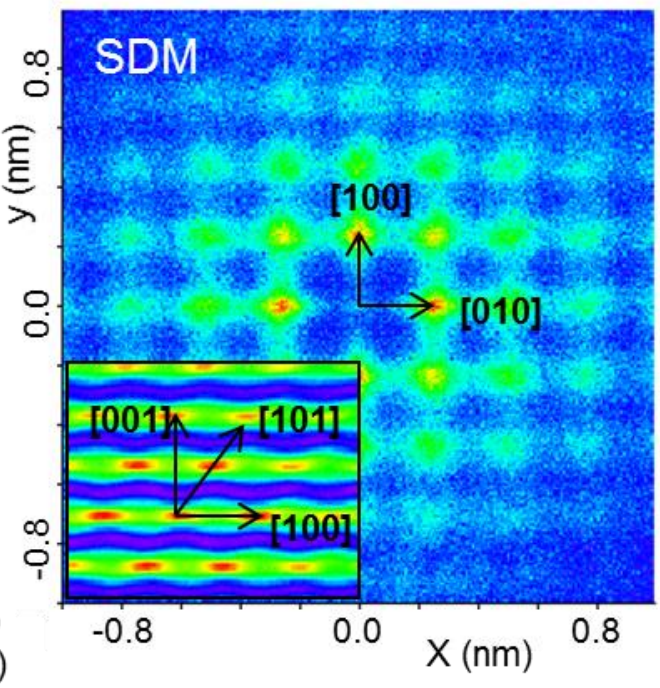

Figure 1. (left) Nickel desorption hit map. (middle) Calculation of ICF by comparison of linear separation with angular separation. (right) SDM showing the reconstructed crystallography in the xyplane and xz-plane (inset).
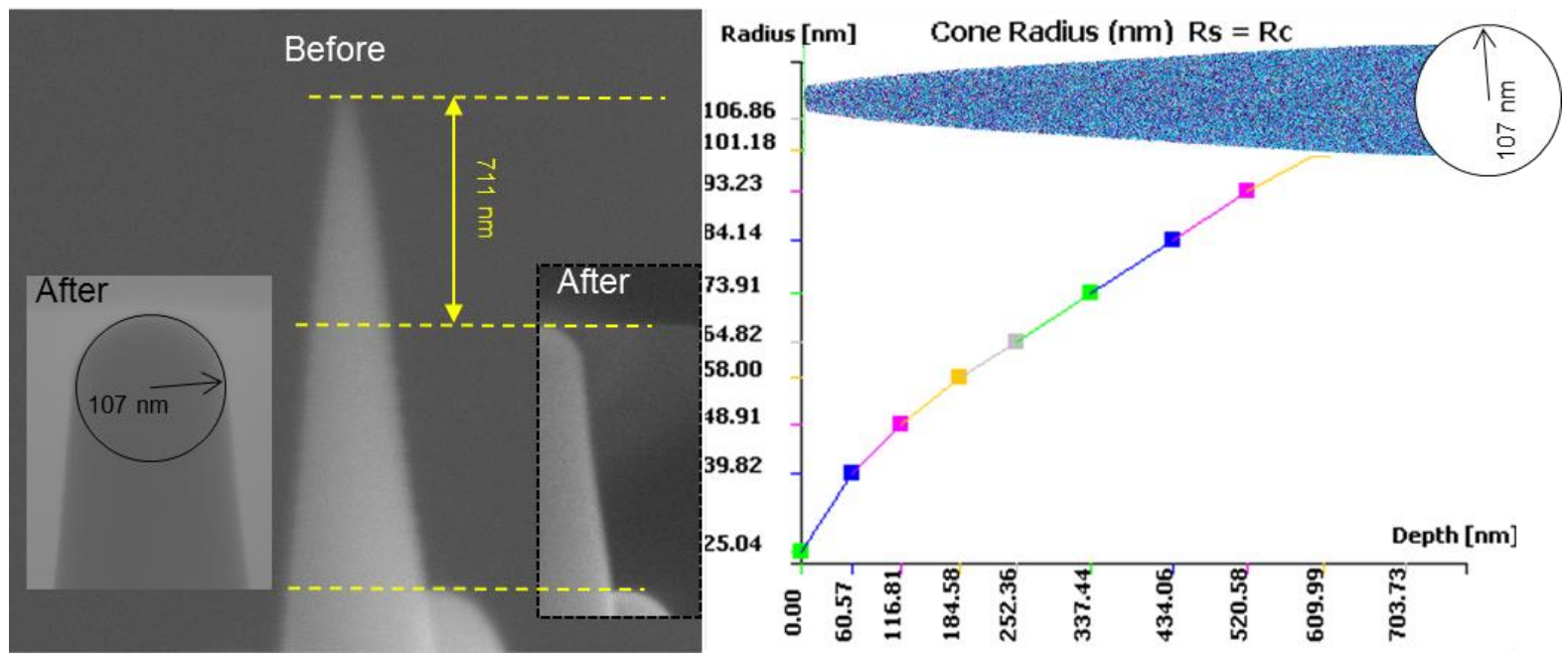

Figure 2. (left) SEM images of prepared tip before and after APT analysis. (right) IVAS tip-profile reconstruction showing the tip diameter measurements and reconstruction with correct final radius (top).

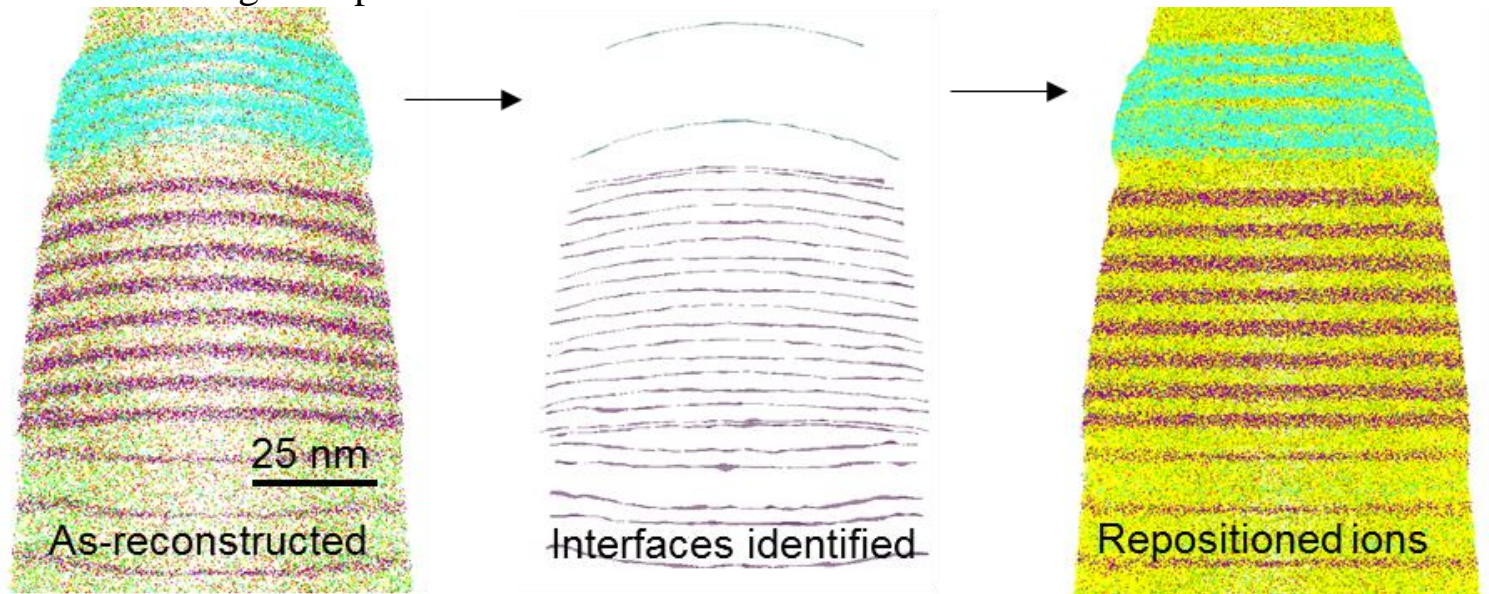

Figure 3. Example of a post-reconstruction correction approach utilizing plane flatness as a metric for correcting ion positions. 